\title{
COVID-19 ARDS: a review of imaging features and overview of mechanical ventilation and its complications
}

\author{
Babina Gosangi ${ }^{1}$ (1) $\cdot$ Ami N. Rubinowitz ${ }^{1} \cdot$ David Irugu $^{2} \cdot$ Christopher Gange $^{1} \cdot$ Anna Bader $^{1} \cdot$ Isabel Cortopassi $^{1}$
}

Received: 28 April 2021 / Accepted: 6 August 2021 / Published online: 26 October 2021

(c) American Society of Emergency Radiology 2021, corrected publication 2021

\begin{abstract}
The first cluster of cases of COVID-19 pneumonia was reported on December 31, 2019. Since then, this disease has spread rapidly across the world, and as of September 17, 2021, there are 226,844,344 cases of COVID-19 worldwide with 4,666,334 deaths related to COVID-19. While most COVID-19 cases are mild, some cases are severe with patients developing acute respiratory distress syndrome (ARDS). The pathophysiology of ARDS includes damage to the alveolar epithelium that leads to increased permeability of the alveolar epithelial barrier causing hyaline membrane formation, interstitial edema, and alveolar edema that results in severe hypoxia. Patients with COVID-19 ARDS are supported by non-invasive or invasive mechanical ventilation with an aim to improve oxygenation and maintain adequate blood oxygen levels. Increased intra-alveolar pressure while on mechanical ventilation may lead to alveolar rupture and thus barotrauma-related injuries such as lung tension cysts, pulmonary interstitial emphysema (PIE), pneumomediastinum, pneumopericardium, and pneumothorax. Recent studies have shown that the rate of barotrauma-related events is higher in patients with COVID-19 ARDS compared to patients with ARDS secondary to other etiologies. Radiologists should be aware of the imaging features of COVID-19 ARDS as well as the complications of mechanical ventilation. This educational manuscript will review the features of COVID-19 ARDS, discuss imaging of patients on mechanical ventilation, and review the imaging features of complications related to mechanical ventilation, including ventilator-associated lung injuries.
\end{abstract}

Keywords Covid-19 ARDS · Endotracheal intubation · Complications of mechanical ventilation · Pneumothorax · Pneumomediastinum $\cdot$ ARDS $\cdot$ Acute respiratory distress syndrome

\section{Introduction}

COVID-19 (Corona Virus Disease 2019) is caused by the corona virus 2 (SARS-CoV-2) [1]. SARS CoV-2 virus gains entry into cells when its spike $(\mathrm{S})$ protein binds to angiotensin converting enzyme 2 (ACE2) receptor [2, $3]$. ACE2 is expressed on the epithelial surfaces of nasal mucosa, oral mucosa, and lungs [2]. The virus causes respiratory infections due to its predilection to involve the lungs [3]. COVID-19 infections are mild in the majority

Babina Gosangi

babina.gosangi@yale.edu

1 Department of Radiology \& Biomedical Imaging, Yale School of Medicine; Thoracic Imaging section, Yale-New Haven Hospital, Yale New Haven Health, New Haven, CT, USA

2 Magnus Hospitals, Hyderabad, India
( $81 \%)$ of the cases; severe disease with hypoxia, dyspnea, involving $>50 \%$ of lung on CT is seen in $14 \%$ of patients; and critical disease with respiratory failure, shock, and multiorgan failure is seen in $5 \%$ of cases $[4,5]$. The rate of critical disease is higher in hospitalized patients with frequencies ranging from 24 to $42 \%$ [6, 7]. Some studies have shown that as high as $42 \%$ of patients with COVID-19 pneumonia could develop acute respiratory distress syndrome (ARDS) with median time to intubation of 8.5 days from the onset of symptoms [7, 8]. COVID-19 ARDS has a worse outcome compared to ARDS related to other conditions with mortality rates ranging from 65.7 to $94 \%$ in patients who require mechanical ventilation $[7,8]$.

In this article, we describe the clinicopathological and radiological findings of COVID-19 ARDS, discuss imaging of patients on mechanical ventilation, and review the imaging features of complications related to mechanical ventilation, including ventilator-related lung injuries. 


\section{COVID-19 ARDS}

COVID-19 ARDS is diagnosed when a patient with confirmed COVID-19 infection develops acute respiratory distress meeting the Berlin 2012 ARDS diagnostic criteria which include (i) acute hypoxemic respiratory failure (ii) developing within 1 week of onset of symptoms (iii) with bilateral air space opacities on X-ray, CT (computed tomography) or ultrasound of the chest (iv) not explained by cardiac failure or fluid overload [9]. COVID-19 ARDS has a slight delay in onset ranging from 8 to 12 days compared to ARDS from other causes where the onset is within 7 days [10]. The lung compliance is normal or high in some patients with COVID19 ARDS unlike ARDS from the other causes [10]. The main cause of COVID-19 ARDS is damage to alveolar epithelial cells resulting in hyaline membrane formation in the initial stages, followed by interstitial edema and fibroblast proliferation $[10,11]$. Imaging studies performed at the time of admission can help in risk stratification of patients with COVID-19 [12]. If diagnosis of ARDS is made at the time of admission, the patient can be directed for intensive care unit (ICU) admission versus in the absence of ARDS patient can be admitted to COVID-19 floor and monitored [12]. Imaging can also help in identifying complications of COVID-19 such as pulmonary embolism, aortic thrombus, and complications arising from mechanical ventilation such as pneumomediastinum, pneumothorax, and superimposed pneumonia [12].

\section{Imaging features of COVID-19 ARDS}

CT findings of COVID-19 pneumonia have been classified as typical, atypical, and indeterminate by Radiological Society of North America (RSNA) expert consensus [13].
Several prior studies have described radiological features of COVID-19, their variation over a period of time, and patient outcomes based on imaging features at the time of presentation [14-17]. We are providing a short summary of the radiological features of COVID-19 pneumonia for the convenience of readers. Imaging features of COVID-19 are classified as typical, which include peripheral ground glass opacities and/or consolidation, peripheral nodular opacities resembling organizing pneumonia, and crazy paving appearance characterized by ground glass opacities with thickened inter and intralobular septae (Fig. 1). Indeterminate imaging features include peribronchial ground glass opacities, and diffuse ground glass opacities (Fig. 2). Atypical imaging features include tree-in-bud opacities, pleural effusions and smooth interlobular septal thickening resembling pulmonary edema, lobar consolidation, and cavitary lesions raising suspicion for other conditions (Fig. 3).

The imaging features of COVID-19 ARDS are similar to ARDS from other etiologies. On chest $\mathrm{x}$-ray, rapid progression of bilateral air space opacities within 7-12 days after the onset on COVID-19 should raise the suspicion for ARDS. There is lower lobe predominance of the air space opacities (Fig. 4) [18]. Similarly, on chest CT rapid progression of lung opacities which involve all 5 lobes in a patient with COVID-19 should also increase the concern for ARDS. Chest CT findings are characterized by anteroposterior gradient of lung opacities [19]. In the acute phase, there is a predilection for dense consolidation to involve the dependent posterior lower lobes with relative sparing of the anterior or non-dependent areas (Fig. 5) [19]. Therefore, prone positioning is thought to be beneficial in these patients to aid in alveolar recruitment and improve lung oxygenation [20,21].

When interpreting chest $\mathrm{x}$-rays in these patients, it is important to be aware of when they are imaged in the prone
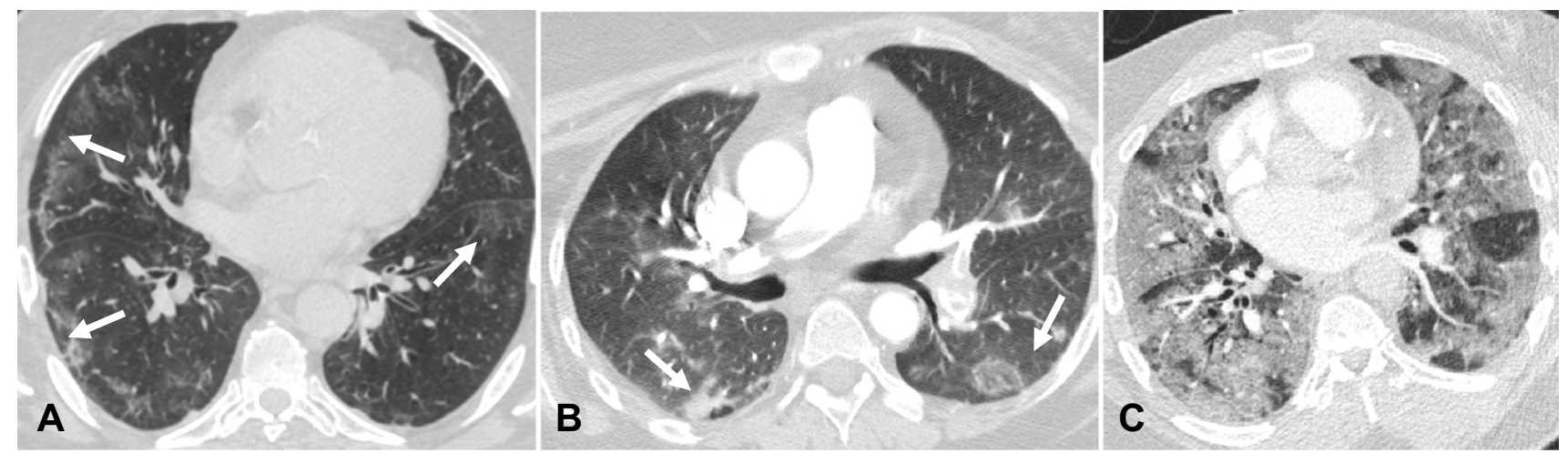

Fig. 1 Chest CT findings showing typical imaging features of COVID-19 pneumonia. A-Axial non-contrast chest CT image in a 48-year-old man with COVID-19 pneumonia demonstrates peripheral ground glass opacities in the bilateral lungs (arrows). B-Axial contrast-enhanced chest CT image in a 68-year-old man with COVID-19 pneumonia shows bilateral peripheral nodular ground glass opacities (arrows). C-Axial contrast-enhanced chest CT image in a 72-yearold woman with COVID-19 pneumonia reveals ground glass opacities in the bilateral lungs with inter- and intralobular septal thickening with a crazy paving appearance 
Fig. 2 Chest CT findings showing indeterminate imaging features of COVID-19 pneumonia. A-Axial contrast-enhanced chest CT image in a 38-year-old man with COVID-19 pneumonia shows bilateral peribronchovascular ground glass opacities (white arrows). B-Axial contrast-enhanced chest CT image in a 60-year-old man with COVID-19 pneumonia reveals diffuse bilateral ground glass opacities
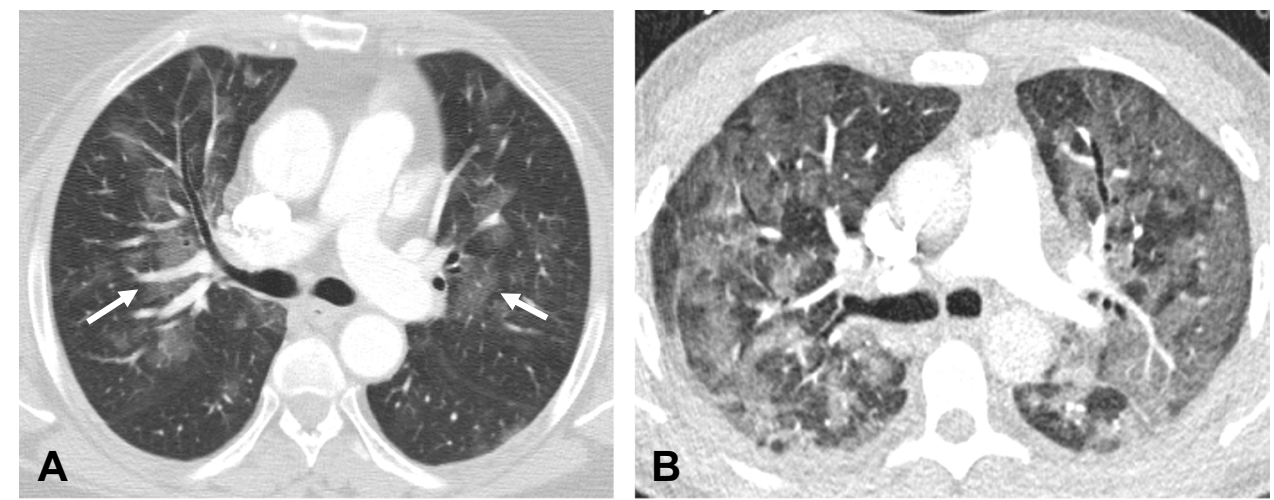

position, as this may artificially cause their air space opacities to appear different when compared with a supine chest x-ray (Fig. 6). Bronchial dilatation can be seen in the nondependent portion of the lungs.
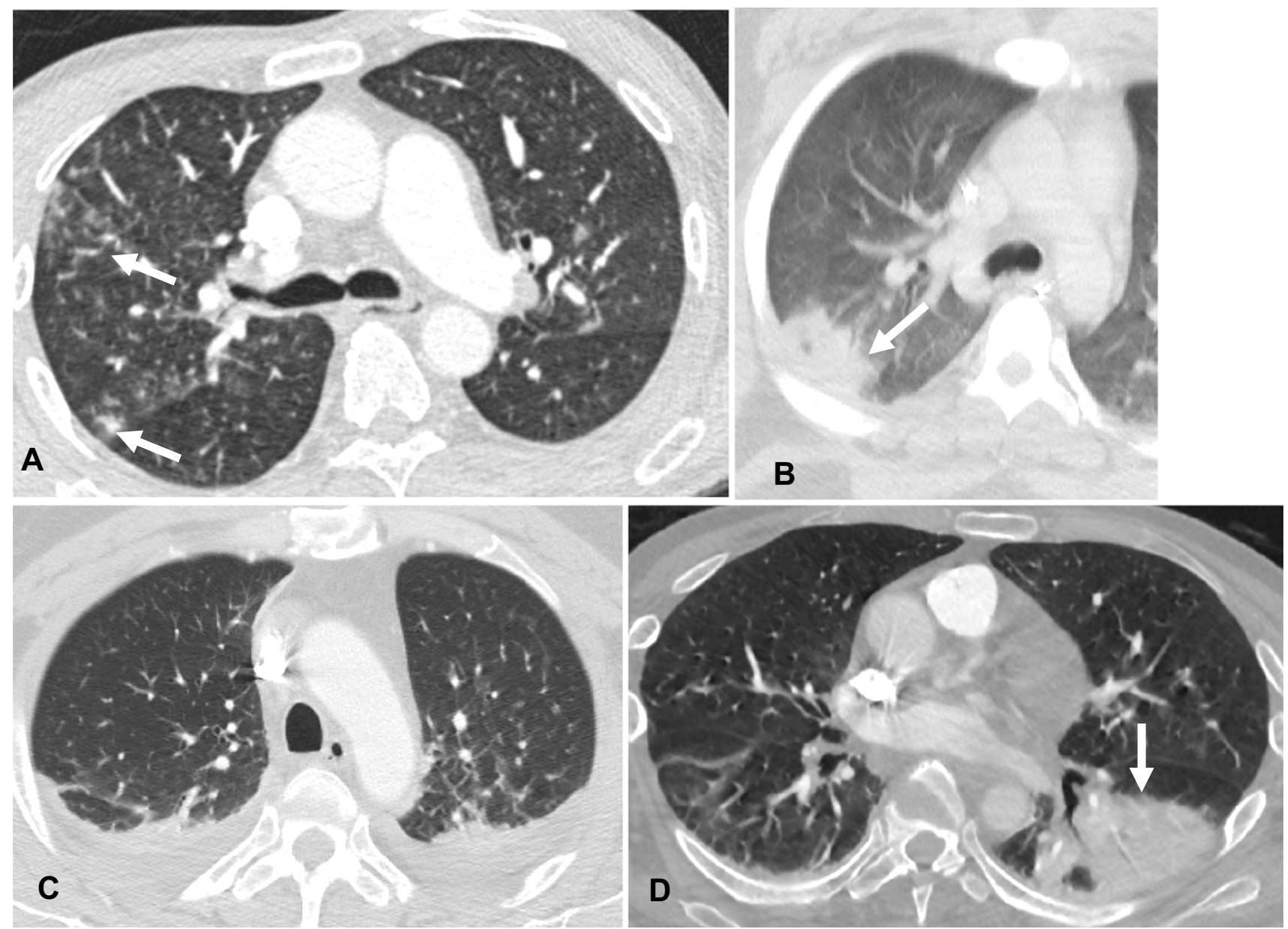

Fig. 3 Chest CT findings showing atypical imaging features of COVID-19 pneumonia. A-Axial contrast-enhanced chest image in a 72-year-old man with RT-PCR test positive for COVID-19 demonstrates clusters of tree-in-bud nodules in the right middle lobe (arrows). B-Contrast-enhanced Axial chest CT image in a 58-yearold man with RT-PCR test positive for COVID-19 reveals a mass-like consolidation in the right lower lobe with a central area of lucency, consistent with a cavitation (arrow). C-Axial contrast-enhanced chest CT image in a 53-year-old man with RT-PCR test positive for COVID-19 shows small bilateral pleural effusions with mild interlobular septal thickening resembling pulmonary edema. D-Axial contrast-enhanced chest CT image in a 66-year-old man with positive RT-PCR for COVID-19 demonstrates consolidation in the left lower lobe (arrow) 


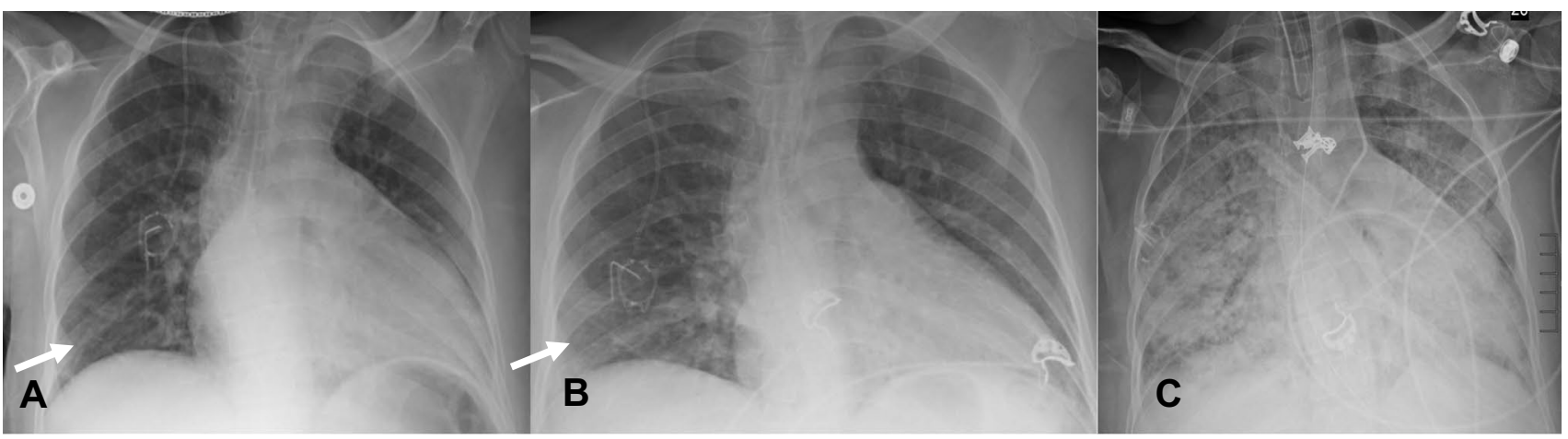

Fig. 4 Serial frontal (AP) chest $\mathrm{x}$-rays in a 64-year-old man showing rapid progression of COVID-19 ARDS A-Initial chest x-ray with faint airspace opacities in the right lower lung (arrow). B-Chest $\mathrm{x}$-ray 2 days later shows increase in the airspace opacities in the right lower lung (white arrow) and retrocardiac opacity. C-Chest $\mathrm{x}$-ray
2 days later (4 days from the initial exam) shows rapid progression of bilateral airspace opacities with a lower lobe predominance and near complete opacification of both the lungs. Also note that the patient is intubated and the presence of a right-sided infusion port
Fibrotic lung changes are seen in some survivors of ARDS with the development of coarse reticulations and traction bronchiectasis in the anterior or non-dependent lungs while the posterior or dependent portions of the lungs are relatively preserved. Therefore, the presence of consolidation seems to protect against later development of fibrosis in patients with ARDS (Fig. 7) [19]. It is important to mention that one must be cautious when interpreting CT images on these patients not to overcall fibrosis while air space opacities are still present since this may have treatment and prognostic indications. Often times what may resemble fibrosis and traction bronchiectasis could potentially be reversible once the air space opacities have resolved. The pathophysiology and features of COVID-19 ARDS are still being studied and its long-term sequelae are yet to be identified.

\section{Management of COVID-19 ARDS}

Low flow nasal oxygen $(<6 \mathrm{~L} / \mathrm{min})$, high flow nasal oxygen (10-20L/min), continuous positive airway pressure (CPAP), and bilevel positive airway pressure (BPAP) are some of the non-invasive techniques used in patients with COVID-19 ARDS that are aimed at improving blood oxygenation while maintaining appropriate $\mathrm{SpO} 2$ levels $[22,23]$. Frequent monitoring of these patients with clinical examination and arterial blood gas evaluation should be carried out every one to two hours. Intubation and mechanical ventilation should be considered when any of the following occur: (i) rapid progression of hypoxemia (ii) no improvement after high flow oxygen administration at 50L/min (iii) increased work of breathing, worsening mental status, hypercapnia, increased tidal volume (iv) hemodynamic instability or multiorgan failure [24].
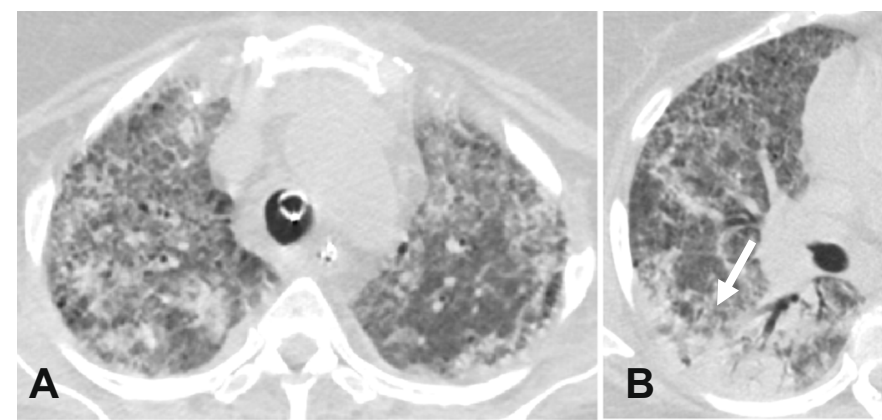

Fig. 5 Axial non-contrast images (A, B, \& C) of the chest CT in a 68-year-old man with COVID-19 pneumonia demonstrate bilateral lung opacities with anteroposterior density gradient. Dense consolidations are seen in the most dependent portion of the lungs (arrows)

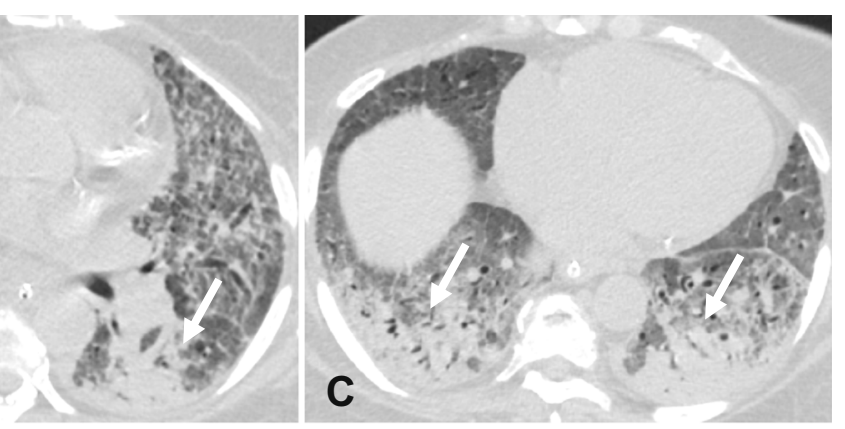

with relative sparing of the more anterior portion of the lungs. While nonspecific, these findings favor COVID-19 ARDS. An endotracheal tube nasogastric tube are also present 
Fig. 6 Frontal chest radiographs in a 64-year-old man with COVID-19 ARDS. A-Supine image of the chest shows dense consolidation in the left lower lung (arrow). B-Image of the chest acquired in prone position in the same patient within two hours demonstrates apparent improvement of consolidations in the left lower lung (arrow) which is positional. A right internal jugular central venous catheter is present
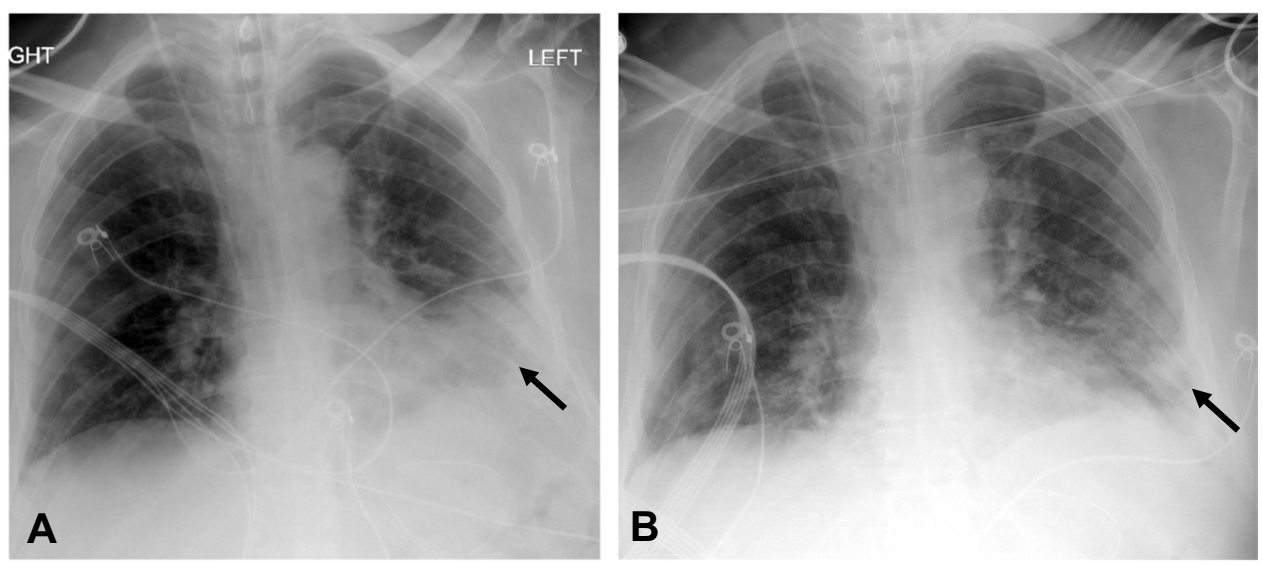

\section{Imaging of patients on mechanical ventilation}

Patients on mechanical ventilation are imaged frequently with chest $\mathrm{x}$-ray, mostly using portable technique at bedside. Imaging helps identify any malpositioned lines and tubes, assessing changes in air space opacities (progression, improvement, or stability), identifying acute superimposed conditions such as volume overload or bacterial infections, and diagnosing ventilator-related complications. Either before these patients require intubation or once they are weaned from the ventilator, it is important to know that it possible to perform chest radiographs with social distancing (at 6 feet) acquiring the images through the glass of the patient's room while maintaining image quality and also preserving personal protective equipment (PPE) [25].

\section{Ventilator-associated lung injury}

Recent study by McGuinness et al. has demonstrated that patients with invasive mechanical ventilation for COVID19 ARDS had higher rate of barotrauma (15\%) compared to non-COVID-19 related ARDS (0.5\%) during the same
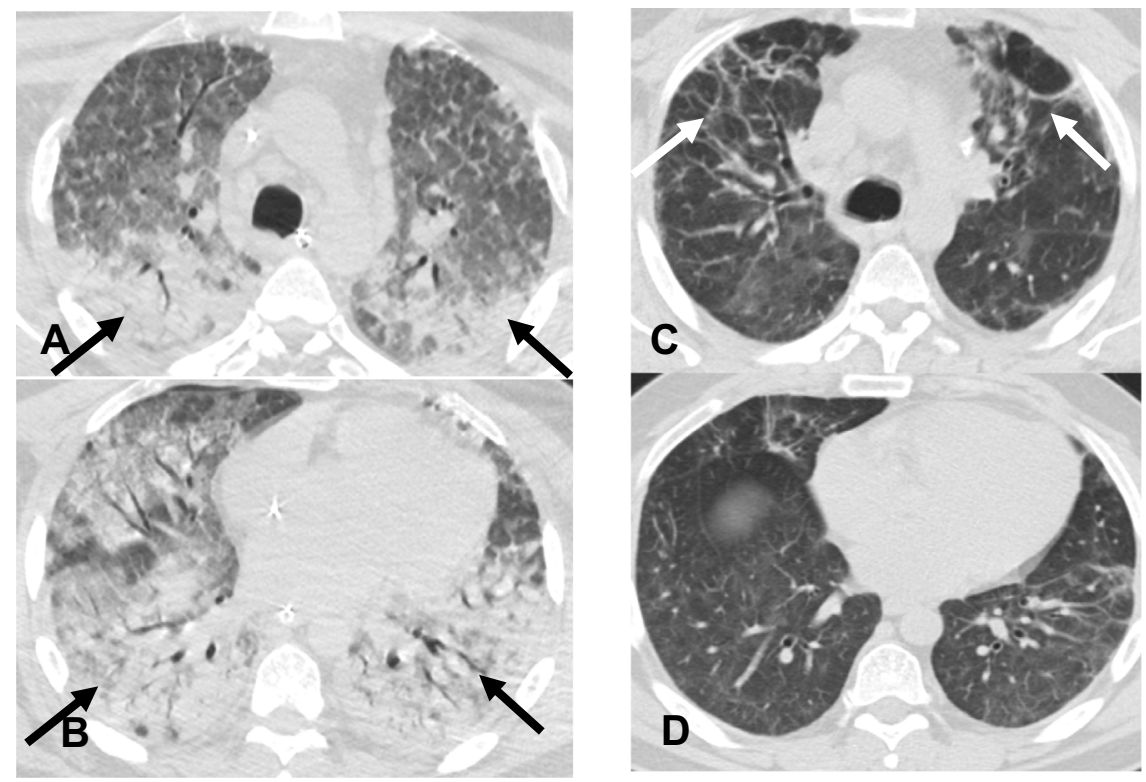

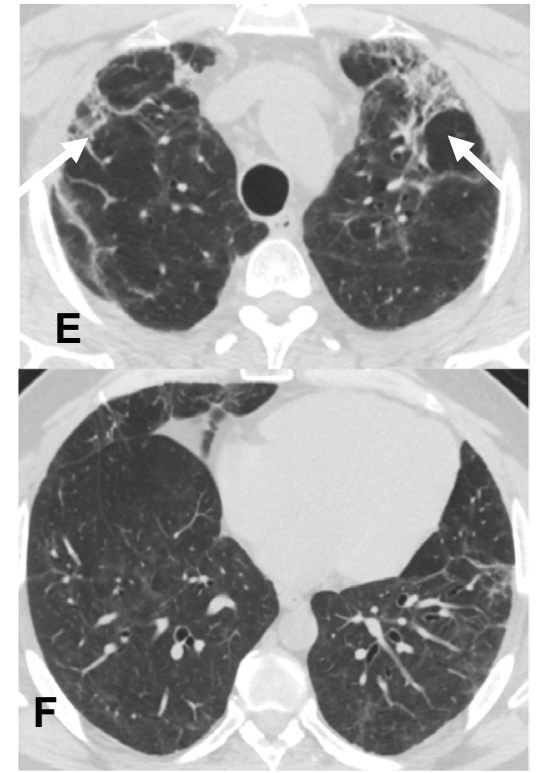

Fig. 7 Axial non-contrast chest CT images in a 44-year-old man with COVID-19 ARDS showing progressive development of fibrosis in the upper lobes with relative sparing of lower lobes. A and B-Initial CT images, show COVID-19 ARDS with dependent consolidations in the bilateral lungs (arrows) and relative sparing of the upper lobes. $\mathrm{C}$ and $\mathrm{D}-\mathrm{CT}$ images from nine months later, show new fibrotic changes with traction bronchiectasis in the bilateral upper lobes (arrows) with improvement of bilateral lower lobe consolidations. E and $\mathrm{F}-\mathrm{CT}$ images eight months later and one year 4 months from initial CT, show peripheral and peribronchial ground glass opacities and consolidations in the upper lobes with traction bronchiectasis (arrows). Lower lobes are spared from fibrotic changes showing that consolidations during ARDS are protective against the subsequent development of lung fibrosis 

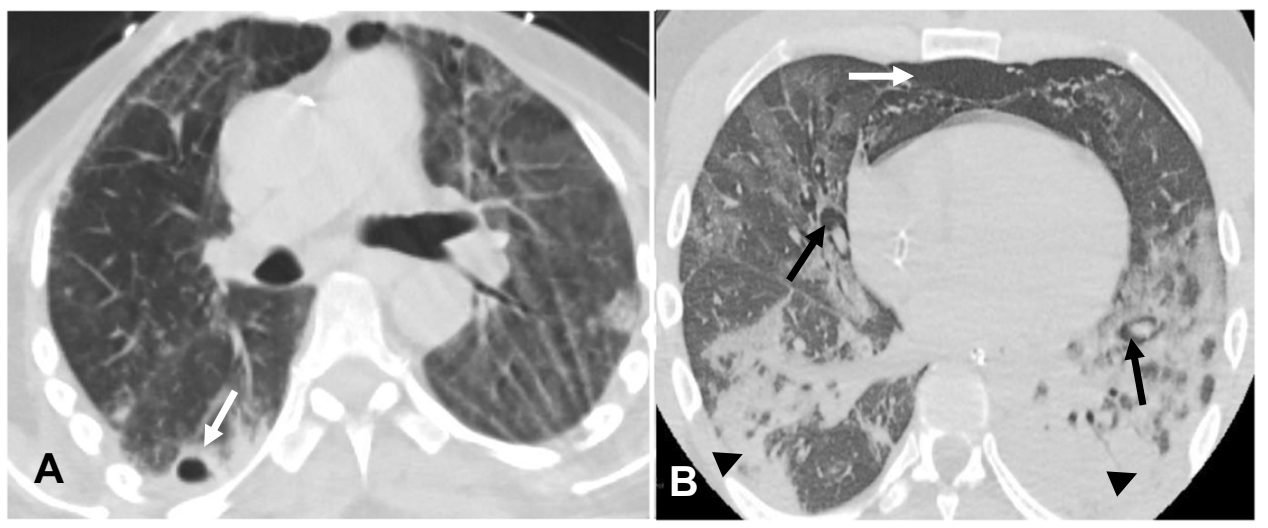

Fig. 8 A-Lung tension cysts. A-Axial non-contrast chest CT image in a 78-year-old man with COVID-19 pneumonia on mechanical ventilation shows cavitary lesion/lung tension cyst in the subpleural right lower lobe (arrow). B-Pulmonary interstitial emphysema. Non-contrast axial chest CT image in a 45-year-old man with COVID-19 ARDS on mechanical ventilation shows pulmonary inter-

time period [26]. Mechanical ventilation causes increased pressure in the alveoli with subsequent disruption of their wall. Barotrauma-related findings include lung tension cysts, pneumomediastinum, pneumopericardium, pulmonary interstitial emphysema (PIE), and pneumothorax. We will review and discuss the imaging features of these entities which can be encountered with ventilator-associated lung injury.

\section{Lung tension cysts}

Lung tension cysts are usually most prominent at the subpleural surfaces and result from rupture of the weaker alveoli along the vessels and interlobular septa [27]. Tension cysts are difficult to identify by chest x-ray. On chest $\mathrm{CT}$, they appear as thin-walled, lucent air-filled spaces more predominant in the anterior part of the upper and middle lobes (Fig. 8A).

\section{Pulmonary interstitial emphysema}

Increased alveolar pressure results in rupture of alveoli with tracking of air along the interstitium, peribronchial tissues, and pulmonary perivascular sheaths [28]. Due to their decreased sensitivity compared with $\mathrm{CT}$, chest $\mathrm{x}$-rays have limited role in identifying PIE. Cystic or reticular lucencies can be seen in the interstitium radiating from the hilum in some cases. Concomitant pneumothorax or pneumomediastinum can sometimes be seen with chest $\mathrm{x}$-ray. On chest $\mathrm{CT}$, linear or dot-like radiolucent air is identified along the bronchi or pulmonary stitial emphysema (PIE). Note the linear lucency surrounding bronchi/ pulmonary artery which is consistent with PIE (black arrows). Air is seen anteriorly consistent with pneumomediastinum (white arrow). Also seen are dependent consolidative opacities in the posterior lower lobes consistent with COVID-19 ARDS (arrowheads)

arteries (Fig. 8B). Reducing the ventilator pressures is the first step towards management of PIE. Pneumothorax and pneumomediastinum can be identified concomitantly.

\section{Pneumomediastinum and pneumopericardium}

Pneumomediastinum has been identified as the most common barotrauma-related event in patients with mechanical ventilation for COVID-19 ARDS [26]. Pneumomediastinum occurs secondary to alveolar rupture with backtracking of air through the interstitium in the peribronchial and perivascular sheaths into the hilum and eventually into the mediastinum, a phenomenon which has been described as Macklin effect $[27,29,30]$. Pneumomediastinum can be identified on chest $\mathrm{x}$-ray by radiolucent air surrounding the normal anatomic structures within the mediastinum. The most common finding of pneumomediastinum is abnormal linear lucency along the left heart border on frontal chest x-ray (Fig. 9) [31]. Air can track from the mediastinum into the neck with development of subcutaneous emphysema. Other signs of pneumomediastinum on chest $\mathrm{x}$-ray are described in Table 1 [31, 32].

On chest CT, pneumomediastinum is visualized as gas outlining the structures within the mediastinum. Air extending into extrapleural space can be differentiated from pneumothorax by the presence of thin connective tissue webs (Fig. 10) [32].

The mechanism of development of pneumopericardium is similar to pneumomediastinum. Isolated pneumopericardium rarely occurs due to mechanical ventilation and is often accompanied by pneumomediastinum [33]. Stretching of medial pleura by the pneumopericardium results in pneumothorax due to rupture of the pleura. On chest x-ray, 

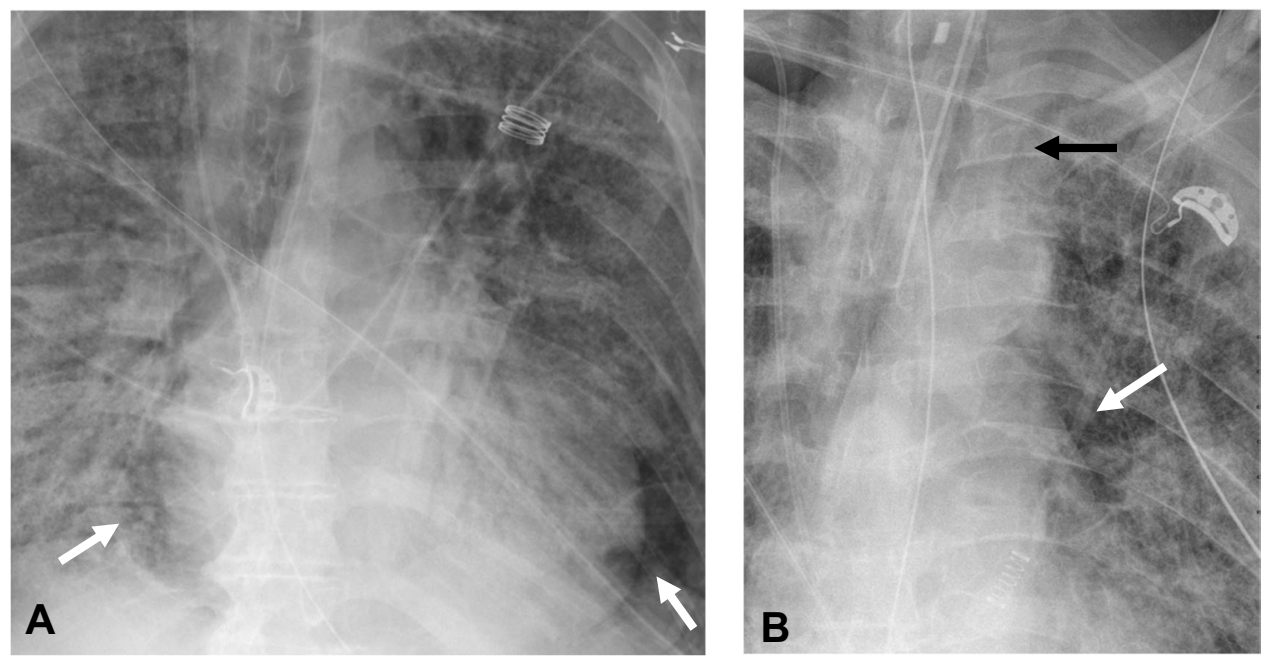

Fig. 9 AP chest radiographs showing pneumomediastinum. AAP chest radiograph in a 72-year-old male patient with COVID-19 ARDS on mechanical ventilation shows lucency surrounding the heart consistent with pneumomediastinum (arrows). B-AP chest radiograph in a 68-year-old man with COVID-19 ARDS on mechani-

air outlines the pericardium as a single band of gas more prominent adjacent to the left ventricle and right atrium, contrary to pneumomediastinum which presents as multiple lucencies of gas in the mediastinum. In pneumopericardium, the gas does not extend into the upper mediastinum and is sharply marginated by the pericardial sac. Pneumomediastinum is self-limited and resolves when ventilatory pressures are reduced. In rare cases when pneumomediastinum is very large, mediastinotomy is performed and a mediastinal drain is placed [34]. cal ventilation shows lucency adjacent to the left heart border (white arrow) and air outlining the lateral aspect of the left subclavian artery (black arrow) consistent with pneumomediastinum. An endotracheal tube, nasogastric tube, and right internal jugular central venous catheter are present

\section{Pneumothorax}

Pneumothorax is the second most commonly identified complication of mechanical ventilation in COVID-19 ARDS accounting for $9 \%$ of barotrauma-related events [26, 35]. In a patient on mechanical ventilation, pneumothorax develops when alveolar pressure increases resulting in rupture of alveoli with air backtracking along the pulmonary interstitium, peribronchial sheaths, perivascular sheaths, and eventually into the pleural space [27]. Chest $x$-ray is readily available to image patients on ventilators and has high specificity for diagnosing pneumothorax, as high as 99\% [36]. However, the sensitivity of chest $\mathrm{x}$-ray in identifying pneumothorax

Table 1 Imaging features of complications of barotrauma

\begin{tabular}{|c|c|}
\hline Complications of barotrauma & Imaging features of complications of barotrauma \\
\hline Lung tension cysts & - Subpleural cysts with a predilection for the upper lobes and right middle lobe \\
\hline Pulmonary interstitial emphysema & $\begin{array}{l}\text { - Cystic or reticular lucencies in the interstitium radiating from the hilum } \\
\text { - Linear or dot-like radiolucent air along the bronchi or pulmonary arteries on CT }\end{array}$ \\
\hline Pneumomediastinum & $\begin{array}{l}\text { - Ring around the artery sign-air surrounding pulmonary artery or its main } \\
\text { branches } \\
\text { - Tubular ring sign-air surrounding major branches of the aorta therefore outlin- } \\
\text { ing both sides of the great vessels } \\
\text { - Double bronchial wall sign-air adjacent to the bronchus creates clear depiction } \\
\text { of the bronchial wall } \\
\text { - Continuous diaphragm sign-air posterior to the pericardium creates continu- } \\
\text { ous contour of the diaphragm } \\
\text { - Extrapleural sign-air extends between the parietal pleura and diaphragm } \\
\text { - Pneumopericardium-air in the anterior pericardium } \\
\text { - Thymic sail sign-elevation of thymus due to air in mediastinum } \\
\text { - Subcutaneous emphysema in the neck }\end{array}$ \\
\hline Pneumothorax & - Thin sharp pleural line with no lung markings beyond the line \\
\hline
\end{tabular}




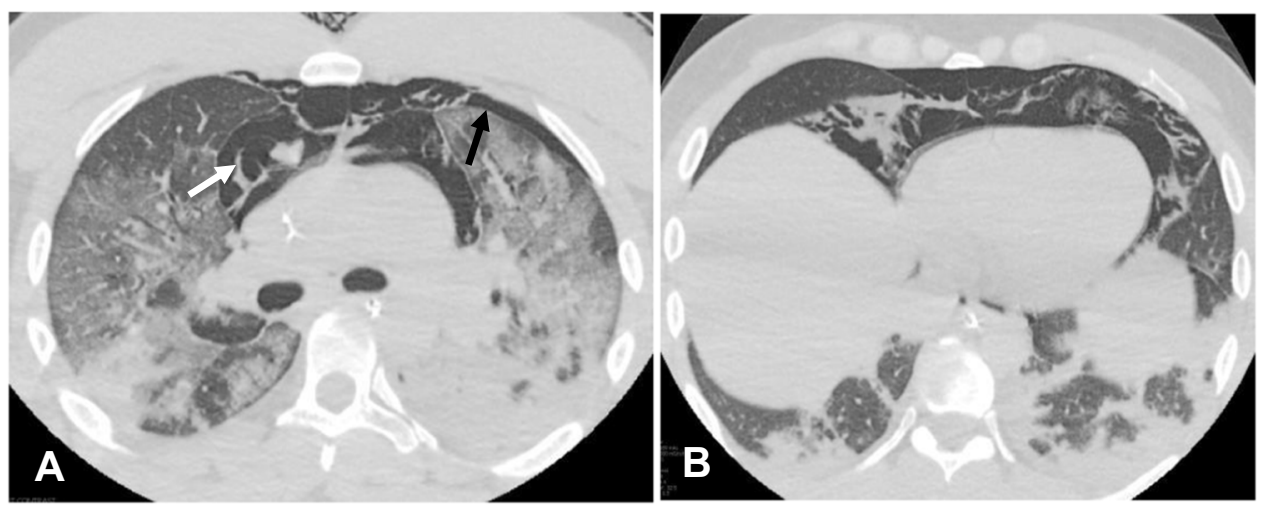

Fig. 10 Axial non-contrast chest CT images in a 72-year-old man with COVID-19 ARDS following mechanical ventilation with pneumomediastinum. A-Air with stranding in the anterior mediastinum consistent with pneumomediastinum (white arrow). Also note small

can be as low as $38 \%$ making it imperative for radiologists to be familiar with all variable radiological appearances of pneumothorax [37]. In the supine position, air commonly rises to the anteromedial pleural space which is the least dependent region and outlines the structures of mediastinum [36]. In the suprahilar region, air outlines the superior vena cava, azygous vein, anterior junction line, and left subclavian vein. In the infrahilar region, air outlines inferior vena cava, heart border, diaphragm under the heart, pericardial fat pad, superior pulmonary vein and causes a medial anterior deep cardiophrenic sulcus [36]. Air accumulating in the subpulmonic space presents with a lateral deep costophrenic sulcus (known as the deep sulcus sign), sharp diaphragmatic outlines, and increased lucency overlying the upper abdomen left pneumothorax (black arrow). B-Air with stranding in the anterior mediastinum consistent with pneumomediastinum. Bilateral ground glass and consolidative opacities are consistent with known COVID-19 ARDS

[36]. When air rises apicolaterally, the visceral pleural line is commonly seen and there is lucency peripheral to the line containing no lung markings [36]. Posteromedial pneumothorax presents as a triangle at the medial costophrenic sulcus with the apex directed towards the hilum [36].

Pneumothorax can be easily identified on lung windows though CT is not routinely recommended as the first modality for the diagnosis of pneumothorax. CT features of pneumothorax are similar to chest $\mathrm{X}$-ray findings with radiolucent air in the pleural space. First-line treatment for patients with pneumothorax is immediate reduction of ventilator pressures and eventually placement of a chest tube if the pneumothorax is large enough or worsens.

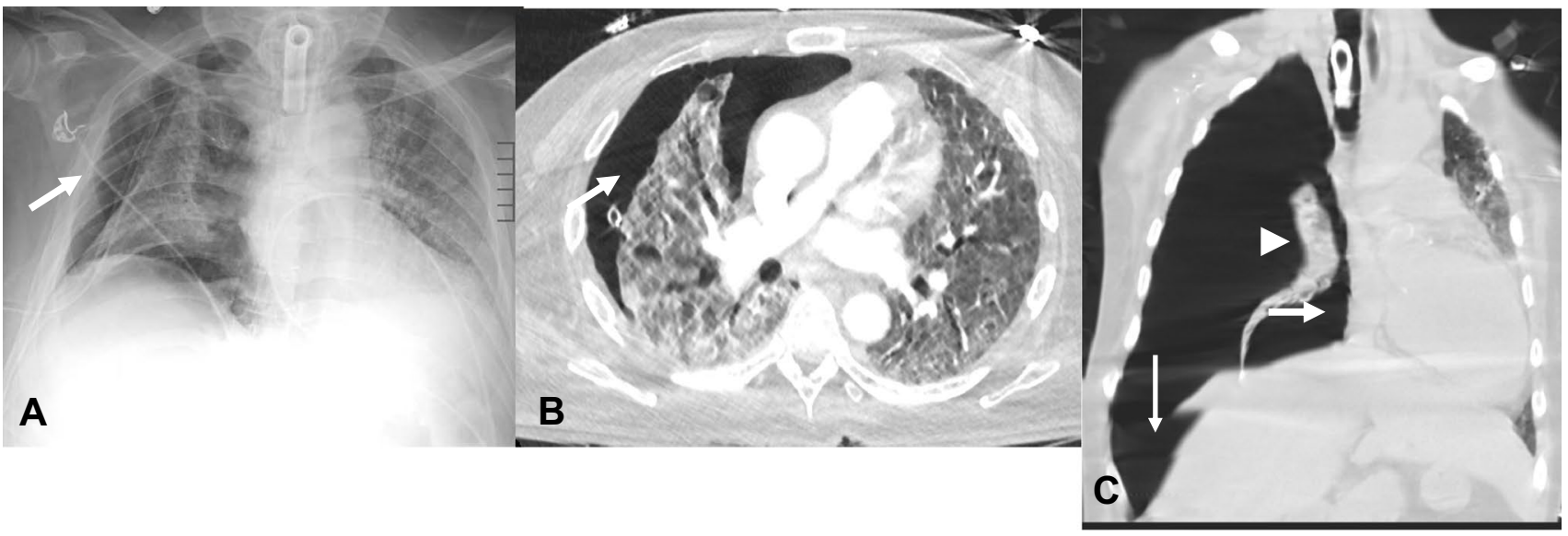

Fig. 11 A-AP Chest x-ray in a 65-year-old man with COVID-19 ARDS on mechanical ventilation shows lucency in the right pleural space consistent with pneumothorax (arrow). A chest tube is present as well. B-Axial contrast-enhanced chest CT image in the same patient shows right pneumothorax (arrow) with chest tube. C-Coronal non-contrast chest CT image in a 55-year-old man with COVID-
19 ARDS on mechanical ventilation shows large right tension pneumothorax with collapse of the right lung (arrowhead), with deviation of the heart and mediastinum to the contralateral side (thick arrow) and flattening of the right hemidiaphragm (thin arrow). A tracheostomy tube is also present in A \& C 


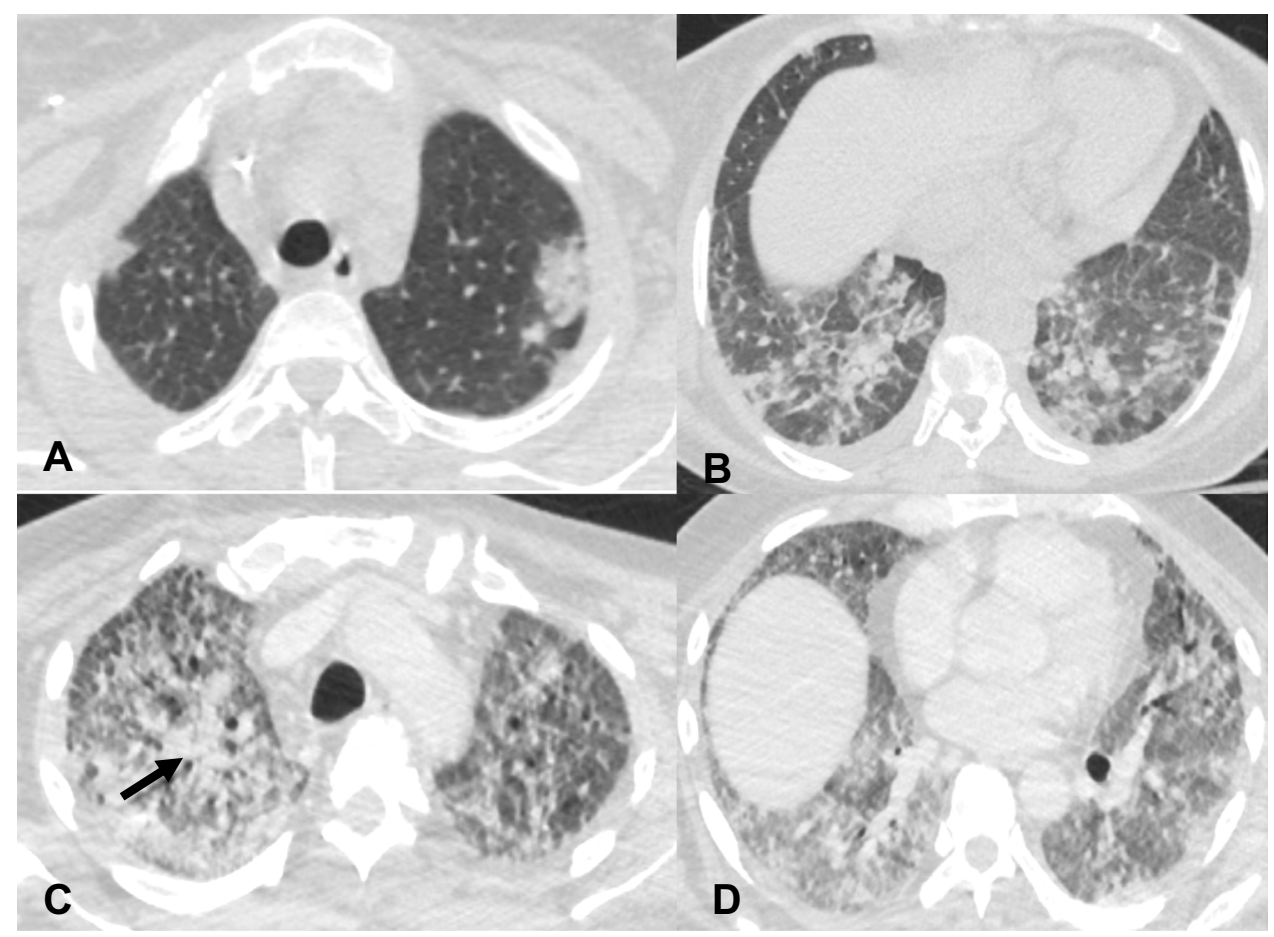

Fig. $12 \mathrm{~A}$ and B-Initial axial non-contrast chest $\mathrm{CT}$ images in a 68-year-old man show ground glass opacities in the left upper lobe and bilateral lower lobes concerning for COVID-19 pneumonia. The patient subsequently developed COVID-19 ARDS and was supported with mechanical ventilation. Axial contrast-enhanced CT images (C and D) were obtained two months later when the patient had increasing secre-

Tension pneumothorax occurs when a pneumothorax increases to the point of causing increased intrathoracic pressure, which limits systemic venous return and decreases cardiac output. Tension pneumothorax is rarely encountered in ARDS because the lungs are stiff, which limits its collapse. While the diagnosis of tension pneumothorax is a clinical tions and worsening fever. There are new consolidations in the right upper lobe (arrow) and worsening ground glass opacities in the lower lobes. Cultures from the endotracheal tube secretions were positive for Klebsiella pneumoniae and Streptococcus pneumoniae, consistent with ventilator associated pneumonia (VAP)

one, findings which are highly suggestive on chest $\mathrm{x}$-ray include a large amount of air in the pleural space with contralateral displacement of the heart and mediastinal structures, ipsilateral flattening or inversion of the diaphragm, and lung collapse (Fig. 11). Tension pneumothorax is a

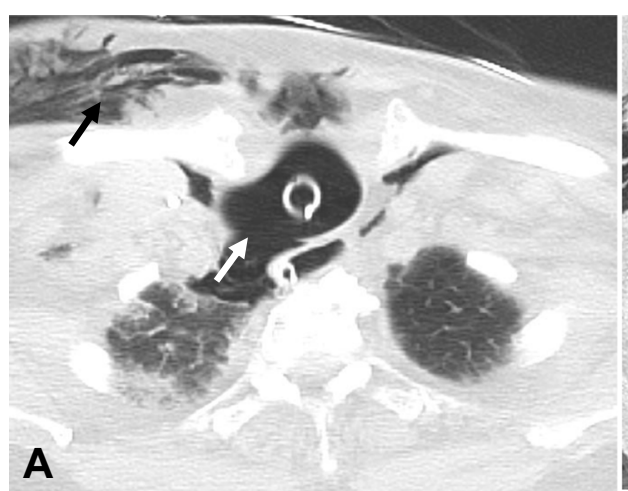

Fig. 13 Axial non-contrast chest CT images in a 94-year old man with tracheal rupture secondary to traumatic intubation. A-There is rupture of the right posterior wall of the trachea (white arrow) with subcutaneous emphysema in the right anterior chest wall (black

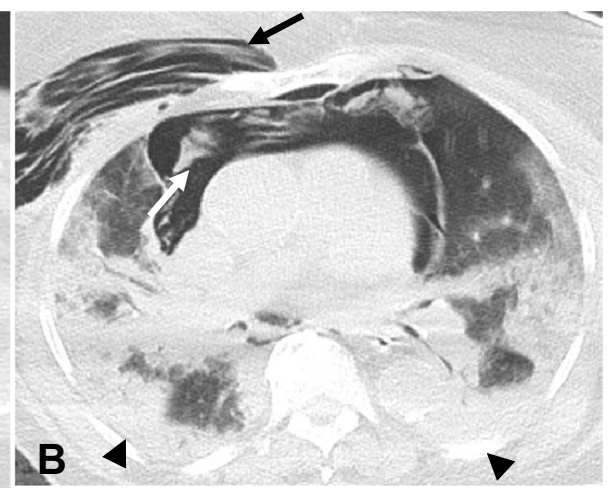

arrow). B-Extensive pneumomediastinum (white arrow) and right anterior chest wall emphysema (black arrow) are also seen. Bilateral consolidative opacities (arrowheads) are also present consistent with COVID-19 ARDS 
life-threatening condition requiring immediate intervention with chest tube placement or needle decompression.

\section{Ventilator-associated pneumonia}

Prolonged hospital stay in COVID-19 patients with mechanical ventilation increases their risk for hospital-acquired infections, particularly ventilator-associated pneumonia (VAP). Diagnosis of VAP is challenging in COVID-19 patients because the clinical features of VAP overlap with worsening COVID-19 pneumonia [38, 39]. Microbiological cultures of deep respiratory secretions or endotracheal aspirate are diagnostic for VAP. The role of imaging remains critical in this scenario. Patients on mechanical ventilation are monitored frequently with chest $\mathrm{x}$-rays. Sudden increase in airspace opacities in COVID-19 patients should raise the suspicion for VAP in patients who are rapidly deteriorating. Chest CT is performed in cases when a patient is clinically deteriorating but the chest $\mathrm{x}$-ray findings are equivocal or stable. Chest CT findings include new superimposed lobar consolidative opacities, increased bilateral consolidative opacities, and/or centrilobular nodules (Fig. 12).

Ventilated patients are also at increased risk of aspiration. Aspiration should be suspected on chest x-ray when there are new or worsening opacities in the lower lobes, commonly seen in the retrocardiac and infrahilar regions. On chest CT, superimposed aspiration is best recognized by centrilobular nodules or consolidative opacities in the dependent parts of the lungs (usually most pronounced in the lower lobes, but can also involve the upper lobes or right middle lobe) when the patient is in the supine position. In patients who are in the prone position, the treein-bud/ centrilobular nodules or consolidations can be seen in bilateral upper lobes and right middle lobe. CT can also demonstrate secretions in the trachea and bronchi in patients with aspiration, as well as a dilated, fluid-filled esophagus or a hiatal hernia, which places the patient at increased risk for aspiration.

\section{Tracheal injury during intubation}

Iatrogenic rupture of the trachea during intubation is extremely rare with an incidence of approximately $0.005 \%$ [40]. The most common site of injury is the posterior membranous wall at the cervical level. The right posterolateral wall is the most common site of injury due to lack of support from the esophagus in this location. Imaging findings on chest radiograph include pneumomediastinum and subcutaneous emphysema in the neck and upper thorax. Findings on CT include the presence of a tracheal diverticulum seen as a small outpouching, most commonly along the right posterolateral wall of the trachea at the level of the thoracic inlet; however, these are much more commonly seen as an incidental finding in patients without history of tracheal injury. Severe cases of tracheal rupture present with discontinuity in the posterior tracheal wall with associated extensive pneumomediastinum, pneumothorax, and subcutaneous emphysema (Fig. 13).

\section{Conclusion}

COVID-19 ARDS results from injury to the alveolar epithelial cells and endothelium resulting in hyaline membrane formation, and accumulation of fluid in the alveolar spaces that results in hypoxemia. Mechanical ventilation is required as a supportive measure in the majority of patients with ARDS. Patients on mechanical ventilation are prone to complications including barotrauma with alveolar rupture and superimposed bacterial pneumonia. Patients with COVID-19 ARDS, as well as patients who are on mechanical ventilation, are imaged frequently; therefore, radiologists play a pivotal role in early and accurate diagnosis. Radiologists should be aware of the imaging features of COVID-19 ARDS and complications of mechanical ventilation, and be ready to alert the clinician when acute, life-threatening ones are present.

\section{Declarations}

Competing interests The authors declare no competing interests.

\section{References}

1. Chan JF-W (2020) A familial cluster of pneumonia associated with the 2019 novel coronavirus indicating person-to-person transmission: a study of a family cluster. Lancet 395(10223):514-523

2. Li F (2016) Structure, function, and evolution of coronavirus spike proteins. Annu Rev Virol 3:237-261

3. Zhou P, Yang X, Wang X, Hu B, Zhang L et al (2020) A pneumonia outbreak associated with a new coronavirus of probable bat origin. Nature 579:270-273

4. Wu Z, McGoogan JM (2020) Characteristics of and Important Lessons From the Coronavirus Disease 2019 (COVID-19) Outbreak in China: summary of a report of 72314 cases from the Chinese center for disease control and prevention. JAMA 323(13):1239-1242. https://doi.org/10.1001/jama.2020.2648

5. Patel A, Jernigan DB, 2019-nCoV CDC Response Team (2020) Initial Public Health Response and Interim Clinical Guidance for the 2019 Novel Coronavirus Outbreak - United States, December 31, 2019-February 4, 2020. MMWR Morb Mortal Wkly Rep 69(5):140-146. https://doi.org/10.15585/mmwr.mm6905e1 
(Erratum in: MMWR Morb Mortal Wkly Rep. 2020 Feb 14;69(6):173)

6. Petrilli CM, Jones SA, Yang J, Rajagopalan H, O'Donnell L, Chernyak Y, Tobin KA, Cerfolio RJ, Francois F, Horwitz LI (2020) Factors associated with hospital admission and critical illness among 5279 people with coronavirus disease 2019 in New York City: prospective cohort study. BMJ 22(369):m1966. https:// doi.org/10.1136/bmj.m1966

7. Wu C, Chen X, Cai Y et al (2020) Risk factors associated with acute respiratory distress syndrome and death in patients with coronavirus disease 2019 pneumonia in Wuhan, China [published correction appears in JAMA Intern Med. 2020 Jul 1;180(7): 1031]. JAMA Intern Med 180(7):934-943. https://doi.org/10.1001/jamai nternmed.2020.0994

8. Puah SH (2020) ATS and APSR Joint Webinar: Global perspectives on COVID-19. https://www.apsresp.org/archive/2020-covid19-webinar.html . Accessed Feb 2021

9. ARDS Definition Task Force, Ranieri VM, Rubenfeld GD, Thompson BT, Ferguson ND, Caldwell E, Fan E, Camporota L, Slutsky AS (2012) Acute respiratory distress syndrome: the Berlin Definition. JAMA 307(23):2526-33. https://doi.org/10.1001/jama. 2012.5669

10. Li X, Ma X (2020) Acute respiratory failure in COVID-19: is it "typical” ARDS? Crit Care 24(1):198. https://doi.org/10.1186/ s13054-020-02911-9

11. Gibson PG, Qin L, Puah SH (2020) COVID-19 acute respiratory distress syndrome (ARDS): clinical features and differences from typical pre-COVID-19 ARDS. Med J Aust 213(2):54-56.e1. https://doi.org/10.5694/mja2.50674

12. Lo Bianco G, Di Pietro S, Mazzuca E, Imburgia A, Tarantino L, Accurso G, Benenati V, Vernuccio F, Bucolo C, Salomone S, Riolo M (2020) Multidisciplinary approach to the diagnosis and in-hospital management of COVID-19 infection: a narrative review. Front Pharmacol 9(11):572168. https://doi.org/10.3389/ fphar.2020.572168

13 Simpson S, Kay FU, Abbara S, Bhalla S, Chung JH, Chung M, Henry TS, Kanne JP, Kligerman S, Ko JP, Litt H (2020) Radiological Society of North America Expert Consensus Statement on Reporting Chest CT Findings Related to COVID-19. Endorsed by the Society of Thoracic Radiology, the American College of Radiology, and RSNA - Secondary Publication. J Thorac Imaging 35(4):219-227. https://doi.org/10.1097/RTI.0000000000000524

14. Pakray A, Walker D, Figacz A, Kilanowski S, Rhodes C, Doshi S, Coffey M (2020) Imaging evaluation of COVID-19 in the emergency department. Emerg Radiol 27(6):579-588. https://doi.org/ 10.1007/s10140-020-01787-0

15. Vernuccio F, Giambelluca D, Cannella R, Lombardo FP, Panzuto F, Midiri M, Cabassa P (2020) Radiographic and chest CT imaging presentation and follow-up of COVID-19 pneumonia: a multicenter experience from an endemic area. Emerg Radiol 27(6):623-632. https://doi.org/10.1007/s10140-020-01817-x

16. Barbosa CS, Chaves GWOG, de Oliveira CV, Bachion GH, Chi CK, Cerri GG, Lima TC, Lee HJ (2020) COVID-19 pneumonia in the emergency department: correlation of initial chest CT findings with short-term outcome. Emerg Radiol 27(6):691-699. https:// doi.org/10.1007/s10140-020-01863-5

17. Mirza-Aghazadeh-Attari M, Zarrintan A, Nezami N, Mohammadi A, Zarrintan A, Mohebbi I, Pirnejad H, Khademvatani K, Ashkavand Z, Forughi P, Arasteh A, Attari JA (2020) Predictors of coronavirus disease 19 (COVID-19) pneumonitis outcome based on computed tomography (CT) imaging obtained prior to hospitalization: a retrospective study. Emerg Radiol 27(6):653-661. https:// doi.org/10.1007/s10140-020-01833-x

18. Jajodia A, Ebner L, Heidinger B, Chaturvedi A, Prosch H (2020) Imaging in corona virus disease 2019 (COVID-19)-A Scoping review. Eur J Radiol Open 11(7):100237. https://doi.org/10. 1016/j.ejro.2020.100237

19. Sheard S, Rao P, Devaraj A (2012) Imaging of acute respiratory distress syndrome. Respir Care 57(4):607-612. https://doi.org/10. 4187/respcare.01731

20. Pan C, Chen L, Lu C, Zhang W, Xia JA, Sklar MC et al (2020) Lung recruitability in COVID-19-associated acute respiratory distress syndrome: a single-center observational study. Am J Respir Crit Care Med 201:1294-1297

21. Guérin C, Reignier J, Richard J-C, Beuret P, Gacouin A, Boulain T, PROSEVA Study Group et al (2013) Prone positioning in severe acute respiratory distress syndrome. N Engl J Med 368:2159-2168

22. Franco C, Facciolongo N, Tonelli R, Dongilli R, Vianello A, Pisani L, Scala R, Malerba M, Carlucci A, Negri EA, Spoladore G, Arcaro G, Tillio PA, Lastoria C, Schifino G, Tabbì L, Guidelli L, Guaraldi G, Ranieri VM, Clini E, Nava S (2020) Feasibility and clinical impact of out-of-ICU noninvasive respiratory support in patients with COVID-19-related pneumonia. Eur Respir J 56(5):2002130. https://doi.org/10.1183/13993003.02130-2020

23. Guy T, Créac'hcadec A, Ricordel C, Salé A, Arnouat B, Bizec JL, Langelot M, Lineau C, Marquette D, Martin F, Lederlin M, Jouneau S (2020) High-flow nasal oxygen: a safe, efficient treatment for COVID-19 patients not in an ICU. Eur Respir J 56(5):2001154. https://doi.org/10.1183/13993003.01154-2020

24. Gattinoni L, Chiumello D, Caironi P, Busana M, Romitti F, Brazzi L, Camporota L (2020) COVID-19 pneumonia: different respiratory treatments for different phenotypes? Intensive Care Med 46(6):1099-1102. https://doi.org/10.1007/s00134-020-06033-2

25. Gange CP, Pahade JK, Cortopassi I, Bader AS, Bokhari J, Hoerner M, Thomas KM, Rubinowitz AN (2020) Social distancing with portable chest radiographs during the COVID-19 pandemic: assessment of radiograph technique and image quality obtained at 6 feet and through glass. Radiol Cardiothorac Imaging 2(6):e200420. https://doi.org/10.1148/ryct.2020200420

26. McGuinness G, Zhan C, Rosenberg N, Azour L, Wickstrom M, Mason DM, Thomas KM, Moore WH (2020) Increased incidence of barotrauma in patients with COVID-19 on invasive mechanical ventilation. Radiology 297(2):E252-E262. https://doi.org/10. 1148/radiol.2020202352

27. Gupta VK, Alkandari BM, Mohammed W, Tobar AM, Abdelmohsen MA (2020) Ventilator associated lung injury in severe COVID-19 pneumonia patients - case reports: ventilator associated lung injury in COVID-19. Eur J Radiol Open 9(8):100310. https://doi.org/10.1016/j.ejro.2020.100310

28. Langlet B, Dournes G, Laurent F (2019) CT features of pulmonary interstitial emphysema. Diagn Interv Imaging 100(12):825-826. https://doi.org/10.1016/j.diii.2019.04.004

29 Brito J, Gregório P, Mariani A, D'ambrosio P, Filho M, Ferreira L, Sawamura M, Pêgo-Fernandes PM (2021) Pneumomediastinum in COVID-19 disease: outcomes and relation to the Macklin effect. Asian Cardiovasc Thorac Ann 29(6):541-548. https://doi.org/10. 1177/02184923211010089

30. López Vega JM, Parra Gordo ML, DiezTascón A, Ossaba VS (2020) Pneumomediastinum and spontaneous pneumothorax as an extrapulmonary complication of COVID-19 disease. Emerg Radiol 27(6):727-730. https://doi.org/10.1007/ s10140-020-01806-0

31 Zylak CM, Standen JR, Barnes GR, Zylak CJ (2000) Pneumomediastinum revisited. Radiographics 20(4):1043-57. https://doi.org/ 10.1148/radiographics.20.4.g00j1131043

32. Bejvan SM, Godwin JD (1996) Pneumomediastinum: old signs and new signs. AJR Am J Roentgenol 166(5):1041-1048. https:// doi.org/10.2214/ajr.166.5.8615238

33. Scacciavillani R, Iannaccone G, Buono MGD, Bello G (2020) Pneumopericardium following mechanical ventilation in 
COVID-19 pneumonia. Eur Heart J Case Rep 4(FI1):1-2. https:// doi.org/10.1093/ehjcr/ytaa213

34 Shennib HF, Barkun AN, Matouk E, Blundell PE (1988) Surgical decompression of a tension pneumomediastinum. A ventilatory complication of status asthmaticus. Chest 93(6):1301-2. https:// doi.org/10.1378/chest.93.6.1301

35. Galetin T, Defosse J, Schieren M, Marks B, Lopez-Pastorini A, Koryllos A, Kosse N, Wappler F, Stoelben E (2020) Sensitivity of chest ultrasound for postoperative pneumothorax in comparison to chest X-ray after lung resecting surgery. Eur J Cardiothorac Surg 57(5):846-853. https://doi.org/10.1093/ejcts/ezz332

36. Tocino IM, Miller MH, Fairfax WR (1985) Distribution of pneumothorax in the supine and semirecumbent critically ill adult. AJR Am J Roentgenol 144(5):901-905. https://doi.org/10.2214/ ajr.144.5.901

37 Akdogan RE, Mohammed T, Syeda A, Jiwa N, Ibrahim O, Mutneja R (2021) Pneumothorax in mechanically ventilated patients with COVID-19 Infection. Case Rep Crit Care 2021:8. https://doi. org/10.1155/2021/6657533 (Article ID 6657533)
38. Moretti M, Van Laethem J, Minini A, Pierard D, Malbrain MLNG (2021) Ventilator-associated bacterial pneumonia in coronavirus 2019 disease, a retrospective monocentric cohort study. J Infect Chemother 27(6):826-833. https://doi.org/10.1016/j.jiac.2021.01. 011

39. Póvoa HCC, Chianca GC, Iorio NLPP (2020) COVID-19: an alert to ventilator-associated bacterial pneumonia. Infect Dis Ther 9(3):417-420. https://doi.org/10.1007/s40121-020-00306-5

40 Borasio P, Ardissone F, Chiampo G (1997) Post-intubation tracheal rupture. A report on ten cases. Eur J Cardiothorac Surg 12(1):98-100. https://doi.org/10.1016/s1010-7940(97)00111-5

Publisher's note Springer Nature remains neutral with regard to jurisdictional claims in published maps and institutional affiliations. 